\title{
Cost-effectiveness of drug monitoring of anti-TNF therapy in inflammatory bowel disease and rheumatoid arthritis: a systematic review
}

\author{
Laura Martelli $^{1} \cdot$ Pablo Olivera $^{1} \cdot$ Xavier Roblin $^{2} \cdot$ Alain Attar $^{3} \cdot$ Laurent Peyrin-Biroulet $^{4}$
}

Received: 7 September 2016/ Accepted: 12 September 2016/Published online: 24 September 2016

(c) Japanese Society of Gastroenterology 2016

\begin{abstract}
Background Therapeutic drug monitoring (TDM) of antiTNF is increasingly used to manage inflammatory bowel diseases (IBD) and rheumatoid arthritis (RA). The costeffectiveness of this strategy is debated.

Methods All studies comparing the cost-effectiveness of a TDM-based strategy and an empirical dose management of anti-TNF in IBD or RA were screened. Studies were identified through the MEDLINE electronic database (up to July 2016), and annual international meeting abstracts were also manually reviewed.

Results Seven studies were included: two randomized controlled trials (RCTs) enrolling 332 patients [247 Crohn's disease (CD) and 85 ulcerative colitis (UC)] and five modeling approaches. Four studies included only CD patients, one included both CD and UC patients, and two included only RA patients. Three studies compared the cost-effectiveness of the two strategies in patients with secondary infliximab (IFX) failure (dose-escalation strategy), one in patients in remission on optimized IFX (deescalation strategy), one in patients starting adalimumab,
\end{abstract}

Laurent Peyrin-Biroulet

peyrinbiroulet@gmail.com

1 Department of Hepato-Gastroenterology, University Hospital of Nancy, Université de Lorraine, Vandoeuvre-lès-Nancy, France

2 Department of Gastroenterology, University Hospital of Saint Etienne, Saint-Étienne, France

3 Department of Gastroenterology, IBD, Nutritional Support, Beaujon Hospital, Clichy, France

4 Inserm U954 and Department of Hepato-Gastroenterology, University Hospital of Nancy-Brabois, université de Lorraine, Allée du Morvan, 54511 Vandoeuvre-lès-Nancy, France and two in patients with clinical response to maintenance anti-TNF therapy. The two RCTs demonstrated that a TDM strategy led to major cost savings, ranging from 28 to $34 \%$. The three modeling approaches with regard to CD patients demonstrated cost savings ranging from $\$ 5396$ over a 1-year period to $€ 13,130$ per patient at 5 years of follow-up. A TDM strategy also led to major cost savings in the two modeling approaches in RA patients.

Conclusions Available evidence indicates that a TDM strategy leads to major cost savings related to anti-TNF therapy in both IBD and RA patients, with no negative impact on efficacy.

Keywords Cost savings · Trough concentrations · Infliximab - Inflammatory bowel disease $\cdot$ Rheumatoid arthritis

\section{Introduction}

The monoclonal antibodies against tumor necrosis factor (anti-TNF)—infliximab (IFX) and adalimumab-are increasingly used to treat patients with IBD and RA who are refractory to standard medications $[1,2]$.

Despite its proven efficacy, up to $60 \%$ of patients who have an initial response to anti-TNF therapy will then experience secondary loss of response, requiring dose escalation or a switch to another anti-TNF to recapture response [3, 4]. Loss of clinical benefit can be due to an increased clearance of the drug in the presence or absence of anti-drug antibodies (ADAs) [5-7].

Cohort studies and post hoc analyses show that serum IFX trough concentrations (TC) correlate with clinical response, clinical remission, and mucosal healing in patients with IBD [8-11]. 
In addition, physicians must determine the best way to de-escalade treatment in patients who have been previously optimized after a dose-escalation strategy and are in sustained remission [12-14].

In the COIN study, healthcare costs in IBD were driven mainly by medication costs, most importantly by anti-TNF therapy, while hospitalization and surgery accounted for only a minor part of healthcare costs [15].

Although TC is related to a drug's efficacy and can be measured adequately, therapeutic drug monitoring (TDM) is not systematically incorporated into daily practice [16]. The systematic use of TDM and the detection of ADAs could be potentially beneficial and economically justified, especially given the high cost of biopharmaceuticals and the complexity of clinical decision-making [17].

The aim of the present article is to review, for the first time, all available studies comparing the cost-effectiveness of an empirical management of anti-TNF treatment versus a test-based strategy in patients with IBD or RA.

\section{Methods}

A literature search was conducted to identify studies that compared the cost-effectiveness of an empirical dose management of anti-TNF therapy and a TDM-based strategy in patients with IBD and RA. We conducted a computerized search of English-language publications listed in the MEDLINE electronic database (up to July 2016). Studies were identified using the following search terms, which were combined: "cost-effectiveness", "cost savings", "costs", "therapeutic drug monitoring", "trough concentrations", "trough levels", "dose-escalation", "deescalation", "tumor necrosis factor-alpha/antagonists and inhibitors", "infliximab", "adalimumab", "inflammatory bowel disease", "Crohn's disease", "ulcerative colitis", "rheumatoid arthritis".

We also manually searched abstracts from the annual international meetings, including the Digestive Disease Week, the American College of Gastroenterology, the United European Gastroenterology Week, and the European Crohn's and Colitis Organization.

We performed a manual selection of studies that satisfied the following inclusion criteria: (1) randomized controlled trials or studies using a modeling approach, (2) enrolment of CD or RA patients treated with an anti-TNF therapy, (3) available data regarding the cost-effectiveness of a TDM strategy compared with empirical dose management of anti-TNF therapy. Eligible articles were then reviewed by the different investigators.

\section{Results}

Among the eligible studies, we identified seven trials comparing the cost-effectiveness of an empirical management of anti-TNF therapy and a TDM-based strategy (Table 1). Two were randomized controlled trials [14, 18], and five used a modeling approach (three used a Markov model [16, 17, 19] and two a discrete event model $[20,22])$. Four studies included only CD patients [18-20, 22], one included both CD and UC patients [14], and two only RA patients [16, 17]. Three studies compared the cost-effectiveness of the two strategies in patients with secondary IFX failure (dose-escalation strategy) [18-20]; one in patients in remission on optimized IFX (de-escalation strategy) [22]; one in patients starting adalimumab therapy [16]; and two in patients with clinical response to maintenance anti-TNF therapy $[14,17]$.

\section{Randomized controlled trials}

Two randomized controlled trials enrolling 332 patients (247 CD and $85 \mathrm{UC}$ ) provided data on the cost-effectiveness of a TDM-based strategy.

A randomized, controlled, single-blind multicenter study was conducted in Denmark [18]. Sixty-nine CD patients with secondary IFX failure were randomized to empirical IFX dose intensification $(5 \mathrm{mg} / \mathrm{kg}$ every 4 weeks) $(n=36)$ or an algorithm strategy $(n=33)$ based on IFX TC and IFX ADAs. Evaluation was performed at week 12, and the primary endpoints were the proportion of patients with clinical response [Crohn's disease activity index (CDAI) decrease $\geq 70$, or $\geq 50 \%$ reduction in active fistulas] and the accumulated costs related to the treatment of $\mathrm{CD}$, expressed as mean cost per patient, based on the Danish National Patient Registry for all hospitalization and outpatient costs in the Danish healthcare sector. This study showed that the costs for intention-to-treat patients were substantially lower $(34 \%)$ for those treated in accordance with the algorithm than by the empirical dose intensification [€6038 vs. €9178 ( $p<0.001)$, respectively] due to discontinuation of treatment because of lack of efficacy in some patients. Efficacy was similar between the two strategies: response rates were 58 and $53 \%$ in the TDM group and in the standard group, respectively $(p=0.81)$. The economic benefit of algorithm-based interventions at IFX failure is maintained throughout 1 year, as demonstrated in another study [21].

A 1-year randomized controlled trial was also conducted in Belgium [14] at a tertiary referral center, including 263 IBD patients (178 CD and 85 UC patients) with stable responses to maintenance IFX therapy. Doses of IFX were adjusted using an algorithm to reach a target TC of 3-7 $\mu \mathrm{g} /$ 


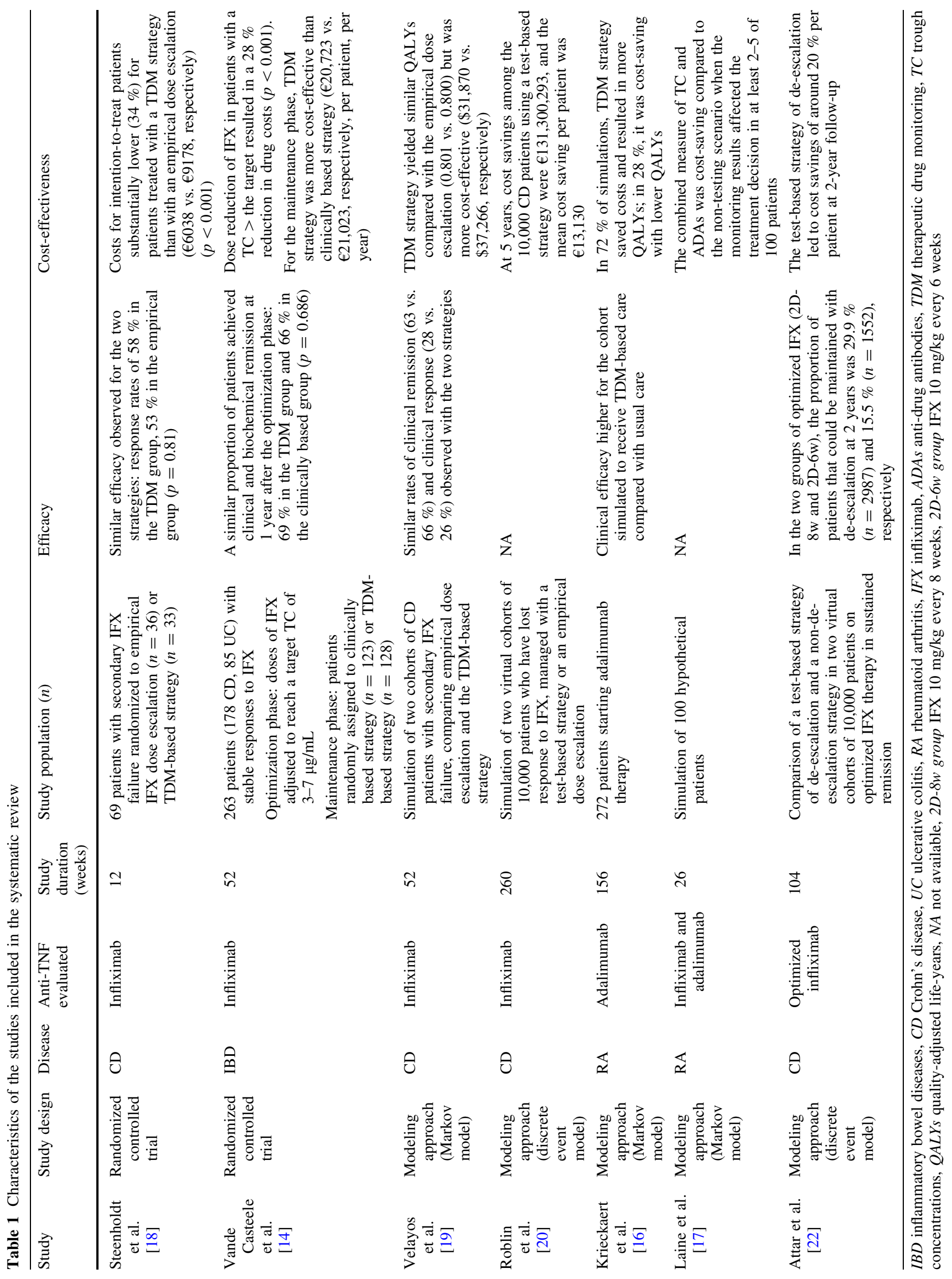


$\mathrm{mL}$ in all patients (optimization phase). Patients were then randomly assigned to receive either IFX dosing based on their clinical features $(n=123)$ or a TDM-based strategy ( $n=128)$ (maintenance phase). The primary endpoint was clinical and biochemical remission at 1 year after the optimization phase. At screening, 115 of the 263 patients had the target IFX TC $(43.7 \%)$. Of the 76 patients with TC $<3 \mu \mathrm{g} / \mathrm{mL}, 69$ patients (91\%) achieved the target TC after dose escalation. This resulted in a higher proportion of $\mathrm{CD}$ patients in remission than before dose escalation (88 vs. $65 \% ; p=0.020)$ and a decrease in the median concentration of C-reactive protein was also observed (3.2 vs. $4.3 \mathrm{mg} / \mathrm{L} ; p<0.001$ ); these changes were not observed in UC patients. Of the 72 patients with TC $>7 \mu \mathrm{g} / \mathrm{mL}, 67$ patients $(93 \%)$ achieved the target TC after dose reduction. This resulted in a $28 \%$ reduction in drug costs from prior to dose reduction $(p<0.001)$. A similar proportion of patients in both groups achieved the primary endpoint: $66 \%$ in the clinically based group and $69 \%$ in the TDMbased group $(p=0.686)$. After dose optimization per patient, a continued TDM-based strategy was not superior to the clinically based strategy for achieving remission after 1 year, but was less expensive ( $€ 20,723$ vs. $€ 21,023$, respectively, per patient, per year).

\section{Studies using a modeling approach}

Five studies with a modeling approach were included in this review: three used a Markov model and two a discrete event model. Three of the studies simulated the cost-effectiveness of the two strategies exclusively in CD patients, and the remaining two in RA patients.

The first study [19] used a decision-analytic model (Markov model) that simulated two cohorts of CD patients with secondary IFX failure, in order to compare the costeffectiveness of an empirical dose-escalation and a testbased strategy over a 1-year period. In the TDM-based strategy, all patients were tested to detect ADAs and quantify IFX TC. Similar rates of clinical remission (63 vs. $66 \%$ ) and clinical response (28 vs. $26 \%$ ) were observed between the two strategies. The TDM-based strategy yielded quality-adjusted life-years (QALYs) similar to those of the empirical dose escalation (0.801 vs. 0.800 , respectively), but was more cost-effective ( $\$ 31,870$ vs. $\$ 37,266$, respectively). The test-based strategy resulted in a higher rate of surgeries (48 vs. $34 \%$ ) and in a lower percentage use of high-dose biological therapy (41 vs. $54 \%$ ).

Another French study [20] used a discrete event model to compare the cost of anti-TNF therapy in two virtual cohorts of 10,000 CD patients with secondary loss of response to IFX: one cohort used a test-based strategy to determine simultaneous ADAs and residual IFX levels, and the other used an empirical dose escalation with up to
5 years of follow-up. Costs were estimated based on the French healthcare system. At 5 years, cost savings among the 10,000 CD patients using a test-based strategy were $€ 131,300,293$, and the mean cost saving per patient was $€ 13,130$. Interestingly, even when including the costs of both postoperative anti-TNF treatment and surgery, dramatic cost savings were still observed: $€ 106,437,792$ for a cohort of 10,000 patients at 5 years. The direct cost of the test had no impact on the results until the cost per test reached $€ 2000$.

The other two studies concerned only RA patients. The first study [16] evaluated the cost-effectiveness of a TDMbased strategy in RA patients starting adalimumab therapy compared with usual care. Disease activity was measured using the disease activity score for 28 joints (DAS28). Treatment response was defined according to the European League Against Rheumatism (EULAR) response criteria. Evaluation was performed at 6 months of adalimumab therapy. In patients responding well to adalimumab and with a low TC $(<5 \mathrm{mg} / \mathrm{L})$, treatment was discontinued. If the target TC $(5-12 \mathrm{mg} / \mathrm{L})$ was reached, adalimumab was continued. A high TC $(>12 \mathrm{mg} / \mathrm{L})$ indicated a reduction in the frequency of adalimumab administration (once in 3 weeks). In patients not achieving EULAR response at 6 months with an appropriate or high TC $(>5 \mathrm{mg} / \mathrm{L})$, a switch to a biological with another mechanism of action was started. If a low TC $(<5 \mathrm{mg} / \mathrm{L})$ was present, mostly due to ADAs, they were switched to a second anti-TNF. Outcomes were simulated using a Markov model, with 3-month cycles, based on a cohort of 272 adalimumabtreated patients with RA over a 3-year period. Costs, clinical effectiveness and QALYs were compared with outcome observed in usual care, and incremental cost-effectiveness ratios were calculated. Clinical effectiveness was higher for the cohort simulated to receive personalized TDM care than for those with the usual care; the average difference in QALYs was 3.84 (95th percentile range -8.39 to 16.20$)$. Testing costs amounted to $€ 10872$. Mean total savings were $€ 2,561,648$ (95th percentile range $-3,252,529$ to $-1,898,087)$, resulting in an incremental cost-effectiveness ratio of $€ 666,500$ or $€ 646,266$ saved per QALY gained from a societal or healthcare perspective, respectively. In $72 \%$ of simulations, personalized care saved costs and resulted in more QALYs; in $28 \%$, it was cost-saving with lower QALYs.

The other study [17] estimated the probabilities of optimal and non-optimal treatment decisions if IFX or adalimumab TC and ADAs were tested or not tested, and modelled the cost-effectiveness of routinely performing TDM. Data on TC and ADA concentrations were obtained from clinically requested monitoring analyses of 486 and 1137 samples from patients on adalimumab and IFX therapy, respectively. TC was within the target range in 
$42 \%$ of samples from adalimumab-treated patients and in $50.4 \%$ of IFX-treated patients. ADAs were detected in approximately 20 and $13.5 \%$ of samples from adalimumab and IFX-treated patients, respectively. ADAs were found in 52.3 and $41.3 \%$ of those with low adalimumab or IFX TC, respectively. The monitoring data were incorporated into probabilities and states in a Markov model for making the optimal treatment decision. The economic impact of clinical decision-making was modeled in a short-term (3-6 months) scenario with 100 hypothetical patients. In this model, the combined measure of TC and ADAs was cost savings compared to the non-testing scenario when the monitoring results affected the treatment decision in at least 2-5 of 100 patients, a proportion which is easily exceeded in real-life clinical practice.

In another, yet unpublished study [22], a discrete event model was used to compare the cost-effectiveness of a testbased strategy of de-escalation versus non-de-escalation strategy in $\mathrm{CD}$ patients in clinical remission under an optimized IFX therapy regime $[10 \mathrm{mg} / \mathrm{kg}$ every 8 weeks (2D-8w group) or $10 \mathrm{mg} / \mathrm{kg}$ every 6 weeks (2D-6w group)]. Two virtual populations of $10,000 \mathrm{CD}$ patients were assigned to management using a test-based strategy or to the current empirical approach of no dose de-escalation. The authors considered three situations of IFX TC for each patient: high TC $(>7 \mu \mathrm{g} / \mathrm{mL})$, therapeutic TC $(3-7 \mu \mathrm{g} / \mathrm{mL})$ and low TC $(<3 \mu \mathrm{g} / \mathrm{mL})$. Dose de-escalation was considered only for patients who had high or therapeutic TC. In the $2 \mathrm{D}-8 \mathrm{w}$ and $2 \mathrm{D}-6 \mathrm{w}$ groups, the proportion of patients that could be maintained with de-escalation at 2 years of follow-up was $29.9 \%(n=2987)$ and $15.5 \%(n=1552)$, respectively. In the 2D-8w group, $93.4 \%(n=4582)$ of the non-de-escalation patients were maintained at a double dose of IFX. The mean difference in terms of cost of treatment for one de-escalation patient versus one non-deescalation patient followed for 2 years was $€ 6830$ (95\% IC 6 434-7226) and $€ 7257$ (95\% IC 6722-7793), respectively. The corresponding mean difference for each 10,000patient $2 \mathrm{D}-8 \mathrm{w}$ and $2 \mathrm{D}-6 \mathrm{w}$ groups were $€ 28$ million and $€ 35$ million, respectively. Cost savings per patient was calculated at around $20 \%$ at 2-year follow-up.

\section{Discussion}

Pharmacokinetics is increasingly used to manage and to optimize anti-TNF therapy in chronic inflammatory diseases, especially in IBD and RA patients. However, the cost-effectiveness of a TDM-based strategy is still debated. We conducted the first systematic review on this topic. All studies included in this systematic review demonstrated the cost-effectiveness of a test-based strategy compared with usual care, with similar efficacy results.
The two randomized controlled trials enrolled 332 patients (247 CD and $85 \mathrm{UC}$ ). The first [18] demonstrated that a TDM-based strategy of dose escalation in CD patients with secondary IFX failure led to cost savings of up to $€ 3140$ at 12 weeks. This effect was mainly due to discontinuation of ineffective treatments in some patients. The two strategies for managing anti-TNF therapy showed similar efficacy. One possible weakness of this study is the fairly short duration of 12 weeks.

The second trial [14], namely TAXIT, demonstrated that less than half of the patients treated with maintenance IFX had optimal IFX TC. Dose escalation in CD patients with suboptimal IFX TC led to a significant increase in patients in clinical remission and a concomitant reduction in CRP concentrations. A similar effect was not seen for UC patients, most likely because the majority of them had a normal CRP at baseline. Dose reduction in CD and UC patients with a supra-optimal IFX TC did not lead to relapse or an increase of CRP, but resulted in significant cost savings $(28 \%)$. Once again, a time frame of 1 year seems fairly short to correctly evaluate long-term clinical efficacy and cost-effectiveness of the two strategies in chronic inflammatory diseases like IBD.

On the other hand, a modeling approach allows the evaluation of a large group of patients, which would otherwise be very difficult to acquire in real life [22], through indirect patient data, thus probably leading to bias.

Changes in a patient's state pose a challenge for physicians in providing the most suitable treatment during the course of IBD. A systems thinking approach is needed when modeling the paths of patients treated for IBD [23]. Different events may occur along a patient's disease course, which can be high in number and can occur randomly from one patient to another. The modeler is then faced with an adaptive complex system in which there are so many possible combinations that methods with the capacity to handle this complexity are needed. For IBD, this complexity makes it difficult to use decision trees, which would be unreadable and unmanageable. Moreover, methods based on differential equations are unusable because of the very nature of the problem. The use of a model-based Markov chain is also not possible, for two main reasons. First, temporal state changes vary from one patient to another. Second, the entire course of the patient must be kept in mind, and this is incompatible with Markov chain modeling. For IBD, we need to use a model based on a discrete event simulation described and computationally modeled by means of the life sequence charts (LSCs), which are an extension of statecharts. In a conceptual framework, the modeler sees the patient as a reactive object whose behavior is characterized by its response to events dispatched from outside its own context and is also affected by its past. Statechart formalism was introduced by Harel's 
team in 1985, and statecharts have since evolved into LSCs. Their use and the use of discrete event simulation appeared in the life sciences in the early 2000s [24], with increasing success. Therefore, the use of a discrete event model is more appropriate than a Markov model. Moreover, variations in the definitions of clinical response across studies and differences in treatment algorithms may have influenced the results yielded by the models.

Velayos et al. [19] used a decision-analytic model (Markov model) that simulated two cohorts of CD patients and compared outcomes for an empirical strategy and a test-based strategy over a 1-year period. In the TDM-based strategy, the authors determined simultaneous ADAs and residual IFX levels to monitor anti-TNF therapy, and analyzed all costs related to CD treatment (surgery, diagnostic tests, health states, etc.). This trial demonstrated the cost-effectiveness of a test-based strategy of dose escalation for managing patients with $\mathrm{CD}$ who have lost responsiveness to IFX. However, no data were reported beyond 1 year for IBD, which is known to be a chronic condition. In addition, a Markov model is not appropriate, because it does not take into account previous states at any given time along the patient's disease course [20].

Roblin et al. [20] used a discrete event model, which seems more appropriate, to compare the cost of anti-TNF therapy in two virtual cohorts of 10,000 CD patients with secondary loss of response to IFX: one using a test-based strategy to determine simultaneous ADAs and residual IFX levels and one an empirical dose escalation up to 5 years of follow-up. Importantly, only the direct costs of anti-TNF therapy in addition to the cost of the test were taken into account. In this study, the TDM-based strategy was associated with major cost savings for anti-TNF therapy: $€ 131,300,293$ for 5 years in a cohort of 10,000 patients. Interestingly, when including the costs of postoperative anti-TNF treatment and of surgery, dramatic cost savings were still observed: $€ 106,437,792$ for a cohort of 10,000 patients at 5 years. Furthermore, the authors found that the cost of the test did not influence the results until it reached $€ 2000$ per test. This result should be taken into account by healthcare authorities when discussing the reimbursement of these tests. However, whether these findings can be extrapolated to other countries will require further investigation. The major limitation of this study is the use of modeling techniques which simplify the real world, but this is the first trial to report cost savings of anti-TNF therapy in patients with $\mathrm{CD}$ using a test-based strategy beyond 1 year in a large cohort of patients.

Another study, which was recently submitted, evaluated the cost-effectiveness of a test-based strategy and an empirical de-escalation of optimized IFX in CD patients in sustained remission [22]. In this discrete event modeling of two large cohorts of 10,000 virtual patients, the authors calculated cost savings per patient of around $20 \%$ at 2-year follow-up. This study demonstrated that de-escalation is an option and is cost-effective when using TDM. However, the study has some weaknesses. First, it used a modeling approach and provided indirect patient data. Second, ADAs were not included in this model, mainly because of the lack of published data or expert experience with regard to dose reduction. Moreover, with the increasing use of IFX biosimilars, treatment costs are coming down, resulting in less cost savings with the TDMbased strategy. Finally, it is unknown whether these data can be extrapolated to other healthcare systems.

Regarding the management of anti-TNF therapy in patients with RA, two studies using a modeling approach have been published. Krieckaert et al. [16] evaluated the cost-effectiveness of personalized treatment of RA using clinical response and serum adalimumab levels [16]. Outcomes were simulated using a patient-level Markov model. Clinical effectiveness was higher for the cohort simulated to receive personalized care than for those receiving usual care, and cost savings on drugs were $€ 2314,354$, while testing costs amounted to $€ 10,872$. However, this study presents some limitations. First, it reflects the situation in the Netherlands and might not be representative for other countries, especially outside Europe. Second, the time frame for analysis was only 3 years, and given the dynamic nature of RA treatment, it is questionable whether the clinical effects of the one-time intervention would persist beyond this time.

The other study [17] estimated the probabilities of optimal and non-optimal treatment decisions if IFX or adalimumab TC and ADAs were tested or not tested, and modelled the cost-effectiveness of routinely performing TDM. Data on TC and ADA concentrations were obtained from clinically requested monitoring analyses. In the model, the combined measure of $\mathrm{TC}$ and ADAs produced cost savings when the monitoring results affected the treatment decision in at least $2-5$ of 100 patients. The main limitation of this study is that laboratory data also contained sera of patients treated with anti-TNF for indications other than RA. Despite these limitations, however, the findings were broadly similar to most of the previously published data from controlled trials with RA patients.

In conclusion, anti-TNF therapy has significantly changed the course of many chronic inflammatory diseases such as IBD and RA. However, the costs of these drugs are high, and their use implies a significant economic burden on healthcare systems.

All seven included studies showed that a test-based strategy with TDM of anti-TNF is more cost-effective than an empirical strategy in both IBD and RA patients, with no negative impact on efficacy. Whether these data can be 
extrapolated to other healthcare systems is yet to be determined.

These findings should be taken into account by physicians and healthcare authorities to guide decision-making in clinical practice and to reduce the costs of healthcare in an era of increasing use of monoclonal antibodies.

\section{Compliance with ethical standards}

Conflict of interest Alain Attar has received lecture and consulting fees from Takeda, Nukleus, AbbVie and Aptalis. Laurent PeyrinBiroulet has received lecture and consulting fees from Merck, AbbVie, Janssen, Genentech, Mitsubishi, Ferring, Norgine, Tillots, Vifor, Therakos, Pharmacosmos, Pilège, BMS, UCB-pharma, Hospira, Celltrion, Takeda, Biogaran, Boehringer-Ingelheim, Lilly, Pfizer, Index Pharmaceuticals, Amgen, Sandoz, Forward Pharma GmbH, Celgene, Biogen, Lycera, Samsung Bioepis and HAC-pharma. Xavier Roblin has received lecture and consulting fees from Merck, AbbVie and Theradiag. The other authors declare that they have no conflict of interest.

\section{References}

1. Peyrin-Biroulet L, Fiorino G, Buisson A, et al. First-line therapy in adult Crohn's disease: who should receive anti-TNF agents? Nat Rev Gastroenterol Hepatol. 2013;10:345-51.

2. Maini RN, Breedveld FC, Kalden JR, et al. Therapeutic efficacy of multiple intravenous infusions of anti-tumor necrosis factor alpha monoclonal antibody combined with low-dose weekly methotrexate in rheumatoid arthritis. Arthritis Rheum. 1998;41:1552-63.

3. Gisbert JP, Panés J. Loss of response and requirement of infliximab dose intensification in Crohn's disease: a review. Am J Gastroenterol. 2009;104:760-7.

4. Arias MT, Vande Casteele N, Vermeire S, et al. A panel to predict long-term outcome of infliximab therapy for patients with ulcerative colitis. Clin Gastroenterol Hepatol Off Clin Pract J Am Gastroenterol Assoc. 2015;13:531-8.

5. Fasanmade AA, Adedokun OJ, Blank M, et al. Pharmacokinetic properties of infliximab in children and adults with Crohn's disease: a retrospective analysis of data from 2 phase III clinical trials. Clin Ther. 2011;33:946-64.

6. Fasanmade AA, Adedokun OJ, Ford J, et al. Population pharmacokinetic analysis of infliximab in patients with ulcerative colitis. Eur J Clin Pharmacol. 2009;65:1211-28.

7. Baert F, Noman M, Vermeire S, et al. Influence of immunogenicity on the long-term efficacy of infliximab in Crohn's disease. N Engl J Med. 2003;348:601-8.

8. Maser EA, Villela R, Silverberg MS, et al. Association of trough serum infliximab to clinical outcome after scheduled maintenance treatment for Crohn's disease. Clin Gastroenterol Hepatol Off Clin Pract J Am Gastroenterol Assoc. 2006;4:1248-54.

9. Seow $\mathrm{CH}$, Newman A, Irwin SP, et al. Trough serum infliximab: a predictive factor of clinical outcome for infliximab treatment in acute ulcerative colitis. Gut. 2010;59:49-54.

10. Adedokun OJ, Sandborn WJ, Feagan BG, et al. Association between serum concentration of infliximab and efficacy in adult patients with ulcerative colitis. Gastroenterology. 2014;147:1296-307.

11. Cornillie F, Hanauer SB, Diamond RH, et al. Postinduction serum infliximab trough level and decrease of C-reactive protein level are associated with durable sustained response to infliximab: a retrospective analysis of the ACCENT I trial. Gut. 2014;63:1721-7.

12. Paul S, Roblin X, Peyrin-Biroulet L. Letter: infliximab de-escalation based on trough levels in patients with inflammatory bowel disease. Aliment Pharmacol Ther. 2015;42:939-40.

13. Baert F, Glorieus E, Reenaers C, et al. Adalimumab dose escalation and dose de-escalation success rate and predictors in a large national cohort of Crohn's patients. J Crohns Colitis. 2013;7:154-60.

14. Vande Casteele N, Ferrante M, Van Assche G, et al. Trough concentrations of infliximab guide dosing for patients with inflammatory bowel disease. Gastroenterology. 2015;148:1320-9.

15. van der Valk ME, Mangen M-JJ, Leenders M, et al. Healthcare costs of inflammatory bowel disease have shifted from hospitalisation and surgery towards anti-TNF $\alpha$ therapy: results from the COIN study. Gut. 2014;63:72-9.

16. Krieckaert CLM, Nair SC, Nurmohamed MT, et al. Personalised treatment using serum drug levels of adalimumab in patients with rheumatoid arthritis: an evaluation of costs and effects. Ann Rheum Dis. 2015;74:361-8.

17. Laine J, Jokiranta TS, Eklund KK, et al. Cost-effectiveness of routine measuring of serum drug concentrations and anti-drug antibodies in treatment of rheumatoid arthritis patients with TNF$\alpha$ blockers. Biol Targets Ther. 2016;10:67-73.

18. Steenholdt $\mathrm{C}$, Brynskov J, Thomsen $\mathrm{O} \varnothing$, et al. Individualised therapy is more cost-effective than dose intensification in patients with Crohn's disease who lose response to anti-TNF treatment: a randomised, controlled trial. Gut. 2014;63:919-27.

19. Velayos FS, Kahn JG, Sandborn WJ, et al. A test-based strategy is more cost effective than empiric dose escalation for patients with Crohn's disease who lose responsiveness to infliximab. Clin Gastroenterol Hepatol Off Clin Pract J Am Gastroenterol Assoc. 2013;11:654-66.

20. Roblin X, Attar A, Lamure M, et al. Cost savings of anti-TNF therapy using a test-based strategy versus an empirical dose escalation in Crohn's disease patients who lose response to infliximab. J Mark Access Health Policy. 2015. doi:10.3402/ jmahp.v3.29229

21. Steenholdt C, Brynskov J, Thomsen $O \emptyset$, et al. Individualized therapy is a long-term cost-effective method compared to dose intensification in Crohn's disease patients failing infliximab. Dig Dis Sci. 2015;60:2762-70.

22. Attar A, Duru G, Roblin X, et al. Test-based versus empirical deescalation strategy of Crohn's disease patients in remission with infliximab optimized treatment: a discrete model event cost savings study (submitted study waiting for acceptance).

23. Sobolev B, Harel D, Vasilakis C, et al. Using the statecharts paradigm for simulation of patient flow in surgical care. Health Care Manag Sci. 2008;11:79-86.

24. Le Lay A, Despiegel N, François C, et al. Can discrete event simulation be of use in modelling major depression? Cost Eff Resour Alloc CE. 2006;4:19. 